Revue d'histoire de l'enfance « irrégulière »

Le Temps de l'histoire

$21 \mid 2019$

L'étrange étrangeté des dossiers de l'enfance irrégulière

\title{
Un des nombreux enfants perdus en Allemagne du fait de la guerre
}

One of the many children lost in Germany because of the war

Jean-Lucien Sanchez

\section{(2) OpenEdition}

Journals

Édition électronique

URL : https://journals.openedition.org/rhei/4681

DOI : 10.4000/rhei.4681

ISSN : 1777-540X

Éditeur

Presses universitaires de Rennes

Édition imprimée

Date de publication : 12 novembre 2019

Pagination : 21-34

ISBN : 978-2-7535-7844-9

ISSN : 1287-2431

Référence électronique

Jean-Lucien Sanchez, "Un des nombreux enfants perdus en Allemagne du fait de la guerre », Revue d'histoire de l'enfance « irrégulière » [En ligne], 21 | 2019, mis en ligne le 12 novembre 2021, consulté le 05 décembre 2022. URL : http://journals.openedition.org/rhei/4681 ; DOI : https://doi.org/10.4000/ rhei.4681

Ce document a été généré automatiquement le 5 décembre 2022

Tous droits réservés 


\title{
Un des nombreux enfants perdus en Allemagne du fait de la guerre
}

One of the many children lost in Germany because of the war

\author{
Jean-Lucien Sanchez
}

1 Les archives versées par le centre pénitentiaire de Fresnes aux archives départementales du Val-de-Marne sont d'une très grande richesse. Parmi celles-ci figurent des dossiers individuels de mineurs détenus à la maison d'Éducation surveillée de Fresnes ${ }^{1}$. L'article 2 de la loi du 5 août 1850 sur l'éducation et le patronage des jeunes détenus prévoyait la création dans les maisons d'arrêt et de justice d'un quartier distinct affecté aux mineurs. À Fresnes, cela se traduisit par l'aménagement en 1929, dans une partie de la troisième division du grand quartier des hommes, d'une maison d'Éducation surveillée pour mineurs ${ }^{2}$. À partir de 1930, ceux-ci furent l'objet d'une procédure d'observation et d'évaluation et un dossier était constitué par des agents de l'établissement sur lequel se basait ensuite le juge pour prononcer sa décision. Ainsi, les mineurs ne restaient que quelques semaines à Fresnes, le temps que leur dossier soit constitué ou qu'ils soient transférés vers un autre établissement. Cette procédure fut ensuite étendue et employée au centre d'observation public de Savigny-sur-Orge. Ouvert au mois de juillet 1945, il était destiné à faire évaluer par un personnel pluridisciplinaire des mineurs qui y demeuraient environ trois mois, le temps qu'y soit établi le même type de dossier ${ }^{3}$.

2 En dépouillant ces dossiers dans le cadre de la préparation d'une exposition pour le Centre d'exposition Enfants en justice, $\mathrm{XIX}^{e}-\mathrm{XX} \mathrm{X}^{e}$ siècle ${ }^{4}, \mathrm{j}$ 'ai été particulièrement ému d'y découvrir ces trajectoires marquées, si jeunes, par l'appareil judiciaire. En tant qu'historien du pénitentiaire, je n'avais travaillé jusque-là que sur l'histoire de condamnés majeurs et il s'agissait donc de ma première incursion dans le domaine de la justice des mineurs. Il m'a ainsi fallu appréhender une législation, des institutions et une bibliographie que je ne maîtrisais guère. Mais ces dossiers m'ont surtout permis d'observer au plus près la genèse du processus d'entrée en délinquance et ses principaux déterminants économiques et sociaux. Ils m'ont également permis, par l'empathie qu'ils suscitent, de me replonger dans cette désormais lointaine période de 
ma vie, l'adolescence, et de me remémorer à quel point la fragilité de l'existence à cet âge peut nous rendre extrêmement vulnérables. Un sentiment d'étrangeté et de malaise s'est emparé de moi en découvrant ces trajectoires judiciaires débutant fréquemment pour la plupart pour des motifs assez bénins, comme un vol de bicyclette, des violences survenues suite à une rixe, un délit de vagabondage, une mésentente entre un fils et son père ayant entraîné une demande de correction paternelle, etc. Ces situations m'ont renvoyé à ma propre adolescence que j'aie passée en partie en Guyane et qui fut marquée par l'échec scolaire et par des actes de rébellion assez similaires à certains de ceux reprochés à ces jeunes délinquants. Ainsi, j'aurais pu peut-être moi aussi connaître ce type de trajectoire si mes parents ne m'avaient pas proposé de poursuivre ma scolarité dans un pensionnat situé en métropole, où je suis parvenu à me reprendre en main. À l'inverse de tous ces adolescents dont les familles (lorsqu'ils en avaient une) étaient en règle générale trop pauvres pour pouvoir leur venir en aide, l'aisance de la mienne m'a ainsi permis d'échapper à un échec scolaire précoce et à son corollaire d'embûches sociales. C'est-à-dire à un déclassement qui ne m'aurait probablement pas permis d'être la personne que je suis devenue aujourd'hui.

Parmi tous les dossiers que j'ai pu consulter, l'un d'entre eux a plus particulièrement retenu mon attention, celui de Jean Gregg, arrêté pour une infraction à la loi sur les étrangers 5 . Une grande partie de ces dossiers font effectivement apparaître des trajectoires marquées par des conflits familiaux (notamment dans les cas de correction paternelle), des enfants élevés par des mères isolées devant assurer seules et avec peu de ressources leur éducation, ou issus de familles violentes ou gravement dysfonctionnelles (abus sexuels, toxicomanie, alcoolisme, etc.). Mais le cas de Jean se distingue nettement de cette typologie et présente un décalage par rapport aux autres dossiers qui m'a d'emblée frappé. La lecture de l'enquête sociale conservée dans son dossier, qui a constitué dans un premier temps la seule pièce qui permette de retracer sa trajectoire malgré les multiples zones d'ombre qu'elle comporte, est fascinante. Et ce même si elle demeure très lacunaire et génère bien plus d'interrogations qu'elle n'apporte de réponses. La découverte de ce matériau a immédiatement suscité en moi l'envie de rétablir le fil de l'histoire de Jean, un peu à l'image, toute proportion gardée, de l'irrésistible attraction qu'a pu opérer pour Patrick Modiano la lecture d'une simple petite annonce contenant l'avis de recherche d'une jeune inconnue ${ }^{6}$. Car cette enquête reflète les contours de la vie d'un adolescent douloureusement marqué par la Seconde Guerre mondiale et qui va connaître un sort pour le moins tragique. Pour tenter d'y échapper, Jean s'acharne à opposer une identité d'emprunt (Gregg n'étant pas son vrai nom) aux différents agents qui cherchent à l'évaluer à la suite de son arrestation. Cette revendication constitue pour lui un mécanisme de défense et la formulation d'une promesse qu'on lui a faite et qui n'a pas été tenue. Mais si son récit est parvenu à dérouter les agents qui cherchaient à le percer à jour, il est également parvenu à me dérouter à mon tour...

\section{La mise en dossier de Jean}

4 Lorsque le juge du tribunal pour enfants de la Seine décrète une ordonnance de placement provisoire contre Jean, le 13 décembre 1947, il est transféré au centre de Savigny-sur-Orge pour y être évalué. Mais en ce qui concerne Jean, la plupart des documents de son dossier n'ont pas pu être établis à Savigny-sur-Orge, car il s'en est 
évadé très peu de temps après son arrivée. N'ont donc été conservés que son enquête sociale, ordonnée par le juge des enfants de Versailles à la suite de sa deuxième arrestation, au mois de novembre 1948, ainsi que son carnet médical établi à la maison d'Éducation surveillée de Fresnes, lorsqu'il y est incarcéré au mois de mars 1949. Il y manque donc de nombreuses autres pièces qui auraient pu apporter beaucoup plus d'éléments susceptibles de mieux nous renseigner sur sa personnalité. Ainsi le seul document qui permette de saisir au plus près l'histoire de Jean demeure son enquête sociale. Car les mineurs placés sous mandat de dépôt doivent obligatoirement faire l'objet d'une enquête sociale effectuée par des déléguées d'associations reconnues d'utilité publique. Cette enquête porte sur: «la famille, l'hérédité, la situation matérielle, la santé, la moralité, l'instruction et le caractère de l'enfant ${ }^{7}$ » et s'achève par une proposition de l'enquêtrice concernant le mineur. Ces enquêtes sont ordonnées par le juge des enfants et une quinzaine de francs est en général allouée aux associations pour couvrir leurs frais. Elles sont très complètes et délivrent énormément de détails sur la vie du mineur, les assistantes sociales prenant le soin d'effectuer une enquête exhaustive en le questionnant lui ainsi que son entourage immédiat (famille, voisins, enseignants, concierges, assistantes sociales, éducateurs, etc.).

\section{Des parents morts dans un camp de concentration}

5 Jean est interrogé par son enquêtrice alors qu'il est hospitalisé à l'hôpital de Versailles depuis le 9 octobre 1948. Il se plaint en effet de souffrir depuis l'âge de cinq ans de brûlures d'estomac. Elles surviennent en moyenne deux heures après chacun de ses repas et il ne peut les soulager qu'en se faisant vomir. Ces malaises se sont aggravés à la suite de son incarcération à la maison d'arrêt de Versailles, le 8 novembre 1948, et ont motivé son hospitalisation pour une bulbite (inflammation du bulbe de l'urètre) et une duodénite (inflammation du duodénum) ${ }^{8}$. Bien que les «médecins traitants croient qu'il s'agit d'un simulateur" ${ }^{9}$ ", son état de santé à son arrivée à Fresnes est caractéristique d'un adolescent qui a souffert de malnutrition et qui a reçu peu ou pas de soins médicaux. Le médecin qui l'ausculte note une «sensibilité à la palpation profonde dans la région épigastrique ", ce qui laisse clairement entendre que Jean ne simulerait donc pas ses douleurs d'estomac. Mais il note également « mouches volantes devant les yeux, sifflements dans les oreilles, amaigrissement, caries dentaires [et] rachitisme assez probable des membres inférieurs ». Autant de symptômes qui révèlent que Jean est « un des nombreux enfants perdus en Allemagne du fait de la guerre ${ }^{10} »$. En effet, bien qu'il lui ait affirmé être américain, son enquêtrice pense plutôt qu'il est né en Allemagne, car il parle couramment cette langue. Elle signale ainsi que son père, d'origine allemande, serait ouvrier électricien, et sa mère, d'origine polonaise, sans profession. Selon ce qu'a pu reconstituer l'assistante sociale, Jean serait parti en 1935 s'installer à Munich avec sa famille. Mais ses parents, Juifs, auraient été arrêtés en 1942 et internés dans un camp de concentration. Ils y seraient morts tous deux en 1944, assassinés ou victimes d'un bombardement. Cette séparation de Jean d'avec ses parents, à l'âge de dix ans, aurait entraîné la fin de sa scolarité et le début de sa longue errance. 


\section{La rencontre avec son « frère d'adoption »}

6 À la suite de l'arrestation de ses parents, Jean aurait été placé dans une ferme en Bavière mais, pris de panique devant l'arrivée des Alliés, il aurait décidé de s'enfuir. Alors «seul au monde", il aurait fait la connaissance au cours de l'année 1945 d'un jeune sous-officier de l'armée américaine, Kevin Gregg. Étudiant de 22 ans, originaire de Californie, celui-ci se serait ému de son sort et efforcé «[de l'] instruire et de [le] protéger ». Jean l'aurait suivi à Biarritz, où Kevin était détaché comme professeur à l'université américaine, puis à Francfort et à Heidelberg au mois de mars ou d'avril 1946.

7 Kevin aurait traité Jean comme un « jeune frère » tant et si bien qu'il aurait décidé de le faire adopter par ses propres parents. Le jeune soldat serait parvenu à se procurer les actes de décès des parents de Jean et aurait entamé une démarche au consulat américain de concert avec ses parents pour pouvoir l'emmener avec lui en Californie. Mais il aurait été rapatrié vers la fin de l'année 1946 avec son régiment aux États-Unis sans être parvenu à faire aboutir la procédure d'adoption. Ses parents auraient bien transmis au consulat américain des garanties financières mais n'auraient pas fourni les documents nécessaires à l'engagement de leur demande d'adoption. Jean n'aurait donc pas pu être adopté et se serait retrouvé à nouveau seul. Il aurait continué néanmoins de correspondre avec la famille Gregg, en espérant pouvoir un jour les rejoindre en Amérique. Jean affirme en outre que Kevin lui aurait "officieusement» donné son patronyme avant de partir et c'est pourquoi il se fait depuis lors appeler « Gregg ».

8 Il aurait séjourné ensuite dans le camp pour personnes déplacées de Niederlahnstein, en Allemagne. Il y aurait fait la connaissance d'un officier français, Robert N., qui lui aurait proposé au mois de janvier 1948 de l'emmener avec lui à Paris où, bien qu'il ne dispose d'aucune pièce d'identité établie à son nom, il lui aurait promis de lui procurer du travail. Jean aurait saisi cette occasion inespérée et aurait gagné Paris. Là, Robert l'aurait hébergé quelques temps chez lui, mais Jean se serait vite rendu compte que son offre était loin d'être dénuée d'intérêt: l'officier lui aurait fait des avances que Jean aurait refusé. Il se serait enfui alors à nouveau. Cette version des faits est toutefois nuancée par les concierges de l'immeuble où réside Robert qui évoquent un mineur "qui se disait américain, paraissait assez hâbleur, mais point méchant ». Selon eux, Jean et Robert se querellaient fréquemment et celui-ci aurait fini par le mettre à la porte de chez lui, affirmant craindre des ennuis avec la Police du fait que Jean ne disposait d'aucun titre de séjour régulier.

9 Suite à cela, il se serait retrouvé à nouveau seul et sans ressources car il lui était impossible de travailler faute de papiers en règle. Il serait revenu alors souvent passer la nuit dans la cage d'escalier de l'immeuble de Robert, ou bien aurait erré dans le quartier de Belleville ou dans celui des Buttes Chaumont, dormant où il le pouvait, dans des caves ou des couloirs d'immeubles, en réussissant parfois à être hébergé et nourri par des gens qu'il serait parvenu à "apitoyer » sur son sort d'orphelin. Au mois de juillet 1948, Jean se serait rendu dans une fête foraine de quartier où il aurait fait la connaissance de Marcelle P., âgée de 17 ans, et de son frère cadet, âgé d'une quinzaine d'années. Jean et Marcelle aurait noué une "amourette toute platonique » et elle lui aurait proposé de l'héberger chez elle, dans une habitation à bon marché (H.B.M.) de la ville de Paris avec ses parents, tous deux ouvriers. Ces derniers auraient accepté de recevoir Jean à la demande de leur fille. Mais le gérant de l'immeuble aurait menacé les 
parents de les dénoncer à la Police pour héberger un étranger dépourvu de titre de séjour régulier. Jean aurait donc été mis à la porte après deux mois passés dans l'appartement, non sans que Marcelle continue à l'aider et à le nourrir à l'insu de ses parents. Épisode qui constituerait donc son troisième et dernier abandon.

\section{L'arrestation, la cavale, la condamnation et l'incarcération}

10 Si les circonstances de son défèrement au tribunal ne peuvent prêter à caution, elles constituent néanmoins autant d'opportunités pour Jean d'alimenter de nouvelles versions de son histoire. Le 11 septembre 1948, il est arrêté par la Police et conduit au commissariat du Pré-Saint-Gervais. Il est coupable d'avoir tenté au cours de la nuit de " soustraire frauduleusement » une automobile, l'enquête ajoutant laconiquement qu'il a été « trouvé de nuit devant la porte d'un garage dans des conditions suspectes ». Il est surtout coupable d'avoir commis une infraction à la loi sur les étrangers puisqu'il a été appréhendé sans titre de séjour régulier. Aux policiers qui l'interrogent, Jean leur délivre la version d'une existence que Kevin lui aurait promise et qu'il a depuis lors plus ou moins idéalisée. Il se déclare ainsi de nationalité américaine, né à Washington et avoir pour parents ceux de son «frère », Kevin Gregg, soldat dans l'armée américaine en garnison à Nuremberg. Il affirme l'avoir suivi à Paris où celui-ci l'aurait laissé seul et où il aurait égaré ses papiers d'identité. Le juge du tribunal pour enfants de la Seine prononce donc le 13 septembre 1948 une ordonnance de placement provisoire à son encontre au centre de Savigny-sur-Orge ${ }^{11}$. Là, il y fait la connaissance de René M., placé pour vol, complicité et recel de vol et que l'enquêtrice dépeint comme " plusieurs fois récidiviste de vol ». Toujours selon l'enquêtrice, René aurait incité Jean à fuguer avec lui afin de participer à un cambriolage. Et le 4 octobre 1948, les deux mineurs prennent la fuite.

11 Dans le signalement émis par le centre de Savigny-sur-Orge et transmis au greffe judiciaire central de Villejuif, on découvre que Jean est désormais nanti d'une profession, celle de "pâtissier », et son prénom est orthographié "John ${ }^{12}$ ». Et que Kevin n'est plus désigné comme son frère, mais qu'il est devenu dans l'intervalle son "père ». Signe que les informations qu'il a transmises à son arrivée au centre demeurent toujours articulées autour de cette identité d'emprunt dont il se serait vu brutalement dépossédé. Les deux mineurs risquent d'être rapidement découverts car ils sont porteurs d'un uniforme fourni par le centre, à savoir un pantalon ainsi qu'une veste marron dite "veste d'aviateur", un chapeau gris "mexicain " et une chemise écossaise rouge et marron à lacets. Enfin, le signalement indique que Jean est fiancé à une certaine "Suzanne" (en fait Marcelle) qui réside dans le $19^{\mathrm{e}}$ arrondissement de Paris, chez ses parents. La cavale de Jean et de René les conduit effectivement le 4 octobre 1948 chez Marcelle. Ils s'y restaurent et Jean échange son uniforme contre des vêtements qu'il avait confiés à son amie. Ils se rendent ensuite à Sèvres, chez la mère de René, mais celle-ci refuse de les recevoir. Deux jours plus tard, soit le 6 octobre 1948, ils se rendent à Saint-Cloud où René, qui connaît bien la ville, a repéré une opportunité de cambriolage. Il s'agit du domicile d'un dentiste où ils parviennent à s'introduire et à dérober 17000 francs, des bijoux, un collier de perles et 300 francs de feuilles d'or. 
12 L'enquête conduite par la préfecture de Police pour retrouver Jean donne dans un premier temps peu de résultats : les diverses vérifications administratives faites à son sujet, notamment au service des garnis et aux sommiers judiciaires, n'aboutissent pas ${ }^{13}$. La Police se rend alors au domicile de Marcelle et apprend par sa mère que Jean aurait été arrêté dans le département de Seine-et-Oise pour un vol et qu'il aurait été mis à la disposition du parquet de Versailles. Elle affirme que cette information lui aurait été fournie par un avocat qui lui aurait adressé une lettre qu'elle aurait ensuite détruite. Et effectivement, vérifications faites, Jean a bien été placé sous mandat de dépôt et écroué à la maison d'arrêt de Versailles le 8 novembre 1948, soit deux jours après avoir commis le cambriolage avec René. Suite à cela, il est condamné le 3 février 1949 par le tribunal pour enfants de Versailles à huit mois de prison pour vol ${ }^{14}$. Jean est incarcéré à la maison d'Éducation surveillée de Fresnes le $1^{\mathrm{er}}$ mai 1949. Il a toutefois été écroué à la maison d'arrêt de Versailles dès le 8 octobre 1948 (date de son mandat de dépôt) avant d'être transféré, le 15 février 1949, au centre pénitentiaire de la Châtaigneraie, situé à La Celle Saint-Cloud, pour y purger sa peine.

\section{Une première conclusion qui n'en est pas une}

13 Le dossier de Jean tel que j'ai pu le consulter dans un premier temps ne permet pas de connaître avec précision les détails de son incarcération. Ni de savoir ce qu'il est devenu après avoir purgé sa peine. Pourtant, plutôt qu'une peine d'emprisonnement, l'assistante sociale conseille dans la conclusion de son enquête qu'il soit remis à la famille Gregg. En effet, selon les renseignements obtenus sur cette famille par le service social correspondant de New York, il s'avère que les Gregg sont non seulement bien réels mais sont aussi d'excellente moralité : le père est ingénieur et la mère est professeure et ils sont «bien établis au point de vue financier ». Ils sont donc "en mesure de subvenir aux besoins du garçon à qui ils semblent attachés ». Les époux Gregg «sont toujours disposés à recevoir le mineur chez eux et correspondent avec lui de façon tout à fait cordiale depuis qu'il est à l'hôpital ». Pourtant ce n'est pas cette solution qui est retenue par le juge des enfants et Jean purge l'intégralité de sa peine de huit mois d'emprisonnement à la maison d'Éducation surveillée de Fresnes, comme l'indique la mention manuscrite « 8 mois prison. Libéré » portée sur la couverture de son carnet médical ouvert à son arrivée dans cet établissement.

\section{Nouveaux rebondissements}

L'histoire aurait pu s'arrêter là et ne se serait sans doute pas écrite au conditionnel si une doctorante de Mathias Gardet, Franciele Becher, qui travaille sur les témoignages d'enfants pendant la Seconde Guerre mondiale, ne s'était pas plongée à son tour dans les dossiers de la maison d'Éducation surveillée de Fresnes. C'est ainsi qu'elle a découvert qu'il existait un dossier établi au nom de Johann ou Jean Schneider, coté 511W 389, qui s'est avéré à la lecture n'être autre que Jean Gregg ${ }^{15}$. Jean est donc retourné en prison, mais sous le nom de Johann S. Et sous cette nouvelle identité (sa véritable semble-t-il cette-fois), c'est une toute nouvelle histoire qui se dessine désormais. Lorsque l'on ouvre ce dossier, on découvre son acte d'état civil qui indique qu'il est né à Munich en 1932. On découvre également que le Jugendamt de Munich signale qu'il est considéré en Allemagne comme un « criminel $^{16}$ » qui a commis 
plusieurs vols et qui s'est évadé de sa prison en tentant d'assassiner un gardien. Et que la Police allemande serait depuis lors à sa recherche. Une note issue d'un courrier adressé vraisemblablement en 1950 par sa mère (qui ne serait donc pas décédée) atténue toutefois ce sévère verdict :

« Nouveaux éléments d'après une lettre de la mère (en allemand). Aurait eu une affaire en Allemagne. Mis dans un centre il aurait assommé un gardien pour s'enfuir. Ce serait pour cela qu'il ne voulait pas rentrer. Aurait déjà changé trois fois d'identité en Allemagne. Se serait fait passer pour Hongrois puis pour Juif. Nombreux mensonges ${ }^{17}$.»

\section{Enrôlement dans les jeunesses hitlériennes}

Dans ce nouveau dossier figure son entretien d'arrivée à Savigny-sur-Orge, en septembre 1948, où il délivre à l'éducateur une histoire particulièrement fantaisiste, mais proche de celle qu'il a déclarée à l'assistante sociale. Il affirme être né à Washington, le 25 mai 1932. Son père serait directeur d'école et sa mère enseignante à Glendale, en Californie. Scolarisé jusqu'au mois de juillet 1944, Johann aurait décidé de quitter l'école pour suivre en Europe le régiment de son frère, Kevin, et aurait débarqué à Cherbourg. Il y aurait travaillé comme pâtissier pour un salaire de 500 francs par semaine. Il aurait ensuite suivi son frère en Allemagne, en Tchécoslovaquie puis à Biarritz, Bayonne, Marseille, Biarritz à nouveau, Francfort, Munich, l'Autriche... Puis le 12 février 1948, le régiment se serait arrêté à Paris. Souhaitant visiter la ville, Johann aurait décidé de ne pas suivre son frère en Allemagne. Nanti de 30000 francs octroyés par Kevin, il se serait rendu chez un ami qu'il aurait rencontré en Allemagne, un certain Robert C., qui l'aurait hébergé contre rémunération. Il aurait fait ensuite la connaissance de Suzanne P., qui vivait chez ses parents. Johann aurait mangé et dormi fréquemment chez elle. Mais le père de Suzanne n'aurait pas semblé voir d'un bon œil cette idylle et aurait refusé que Johann reste sous son toit. À court d'argent, il se serait alors installé sous une tente achetée à un ami et ce serait au cours d'une ballade, transi de froid, qu'il aurait été surpris devant la porte d'un garage par le propriétaire d'une voiture qui l'aurait « regardé avec des yeux méchants ». Johann serait ensuite retourné chez sa fiancée pour y déjeuner et c'est à cette occasion que la Police l'aurait arrêté.

Puis dans un autre entretien d'arrivée à Savigny-sur-Orge, en avril 1950, il se présente sous l'identité de Jean Schneider, né le 25 mai 1930 à Reims et délivre encore une nouvelle version de sa vie, mais qui semble cette fois-ci plus vraisemblable que les précédentes. Ses parents, tous deux allemands et dont il serait sans nouvelles depuis 1944, s'entendaient bien et ne se disputaient jamais selon lui. Son père serait électricien, sa mère femme au foyer, et le couple aurait eu sept enfants. D'après l'acte de naissance versé à son dossier, Johann n'a jamais connu son père biologique, et a été reconnu par son beau-père en 1940. La famille vivait à Khübach, en Bavière : «Je ne sais plus combien il y a avait de pièces. C'était propre chez nous [...] ce n'était pas riche, mais on avait tout ce qu'il fallait ${ }^{18}$. » Johann aurait stoppé sa scolarité pour intégrer les Hitlerjugend (jeunesse hitlériennes), dont l'enrôlement était obligatoire. Bien qu'il affirme s'y être plu, il y aurait commis son premier vol, poussé par la faim selon lui : il aurait subtilisé des tickets d'alimentation dans le bureau du chef du centre. Démasqué, il aurait été envoyé dans un centre à Freising, près de Munich, où il serait resté quelques mois. Puis, profitant de l'arrivée des Alliés, il serait parvenu à se sauver (en assommant un gardien?) et aurait rejoint un régiment américain dans lequel il serait 
resté trois ans. S'en serait ensuite suivi l'épisode de sa condamnation et de son incarcération à Fresnes.

\section{Un déséquilibré psychique à note perverse}

17 Libéré de Fresnes le 15 février 1949, Johann est aidé par une assistante sociale et est employé au Lido pendant 15 jours. Tombé malade, il est envoyé à la campagne, à Précysous-Thil, en Côte d'Or. Mais il se serait laissé une nouvelle fois entraîner dans un vol, celui d'un médecin, par un «gars» dont il ne sait pas le nom... Arrêté trois semaines plus tard, il est condamné par le tribunal correctionnel de Dijon à six mois de prison. À sa sortie, il est embauché par un libraire parisien et décide de se rendre à la préfecture de Police pour y obtenir des papiers d'identité. C'est alors qu'il est à nouveau arrêté pour infraction à la loi sur les étrangers et condamné en avril 1950 à six mois de prison. À l'éducateur qui l'interroge à son arrivée à Savigny-sur-Orge, Johann affirme qu'il souhaite retourner en Allemagne pour y retrouver une jeune fille dont il serait très épris, Ingrid. Il l'aurait rencontrée lors de son passage dans le régiment américain et semble très amoureux d'elle si l'on en croit les nombreuses lettres d'amour qui parsèment les six cahiers que contient son dossier, intégralement rédigés en allemand. En définitive, chez ce "garçon qui ment pour cacher quelque chose ", il semblerait que le départ d'Allemagne et l'errance qui s'en serait suivie aient essentiellement été motivés par sa mésentente avec sa mère :

«Je ne voudrais pas retourner avec ma mère. Quand je me suis évadé du centre en Allemagne, je n'ai pas été chez elle car j'y étais malheureux. Mon père m'aimait bien et était gentil. Mais ma mère me battait tout le temps... elle me crachait dans la figure. Elle ne touchait pas aux autres frères et sœurs. Pourtant j'obéissais bien, je ne lui répondais pas, je travaillais mais elle ne m'aimait pas. Ce que je voudrais c'est avoir des papiers pour aller en Amérique chez les gens qui veulent m'adopter. [...] Avant d'aller en Amérique, je veux retourner en Allemagne pour voir la jeune fille, $\mathrm{M}^{\text {lle }}$ Ingrid [...]. Si je pouvais me marier avec elle, on vivrait ensemble en Amérique $^{19}$. "

Cette nouvelle version des faits ne semble toutefois pas convaincre le docteur Mâle chargé de réaliser son examen psychiatrique à Savigny-sur-Orge et qui évalue son âge mental à celui d'un enfant de onze ans :

«Ce sujet se présente comme un déséquilibré psychique à note perverse, chez lequel domine l'instabilité migratrice, la fabulation (fausses identités successives) s'accompagnant de vols et cambriolages. Allemand déjà en maison de rééducation en 1944 en Allemagne (évasion et violences). Vit avec les troupes américaines pendant plusieurs années, puis en France où il est condamné à deux reprises à des peines de prison, pour vol. Il ne faut pas surestimer l'importance des événements de l'époque. La note constitutionnelle de déséquilibre avec fond névropathique paraît dominante, et s'accompagne de conflits familiaux et d'hostilité vis-à-vis de la mère. [...] Semble devoir, étant donné la sévérité du diagnostic, être renvoyé dans sa famille en Allemagne ${ }^{20}$. »

\section{Une seconde conclusion encore loin d'en être une}

Serait-ce donc ce conflit avec sa mère, que Johann semble tant redouter, qui l'aurait poussé à s'enfuir de chez lui et à inventer la mort de ses parents afin d'éviter son renvoi en Allemagne? Une fois de plus, les différentes versions qu'il livre de sa vie déroutent 
et ne permettent pas, même si beaucoup d'expériences se recoupent d'un dossier à l'autre, de saisir précisément sa trajectoire et ses véritables intentions. D'autant plus que ses récits imaginaires sont très similaires à ceux d'un autre «fils de la Seconde Guerre mondiale » étudié par Guillaume Périssol ${ }^{21}$. En effet, les histoires inventées par Jean-John-Johann sont étonnamment proches de celles imaginées par un autre orphelin de guerre interné également au centre de Savigny-sur-Orge, John-Roman, arrêté pour vagabondage à Paris en 1945. Celui-ci, qui prétend s'appeler John et être né à New-York, ne parle qu'allemand. Habitant Vienne, il affirme que ses deux parents seraient morts pendant la guerre : sa mère aurait été internée dans un camp et fusillée par des SS tandis que son père serait mort au combat. Interné dans un camp de réfugiés à Salzbourg, il y aurait fait la connaissance d'un soldat américain qui lui aurait donné son patronyme (avant de mourir poignardé peu après). Dans une autre version des faits, Roman prétend que sa mère se serait mariée avec un soldat américain qui aurait pris la fuite devant l'avancée des troupes russes (qui auraient également tué ou déporté son père biologique). L'orphelin aurait ensuite accompagné un régiment de soldats américains jusqu'à Paris et souhaiterait rejoindre sa famille qui résiderait aux ÉtatsUnis. Enfin, dans une ultime version des faits qu'il livre à une assistante sociale, il affirme en définitive s'être enfui d'Autriche pour échapper à sa mère qui le battait ! Le récit de Roman est décousu, plus encore que celui de Johann, mais l'étroite ressemblance des faits décrits par les deux adolescents est troublante. Est-ce qu'ils ne se seraient pas inspirés l'un l'autre au cours de leur séjour au centre de Savigny-surOrge où ils ont effectivement cohabité ensemble pendant un mois et demi, du 14 septembre au 4 octobre $1948^{22}$ ? Il demeure difficile de l'affirmer avec certitude. Mais au regard de tous ces rebondissements, je me garderai bien désormais d'avancer une nouvelle hypothèse quant à l'histoire de Johann...

\section{NOTES}

1. Je remercie Véronique Blanchard et Mathias Gardet de m'avoir signalé ce fonds.

2. Cette institution fut instaurée par un décret en date du 31 décembre 1927 qui a transformé les colonies pénitentiaires en maisons d'Éducation surveillée pour les garçons et en écoles de préservation pour les filles. Voir YVOREL Élise, Les enfants de l'ombre: la vie quotidienne des jeunes détenus au XXe siècle en France métropolitaine, Rennes, Presses universitaires de Rennes, 2007, p. 59.

3. BLANCHARD Véronique, "Centre d'exposition "Enfants en justice" ", Revue d'histoire de l'enfance "irrégulière» [En ligne], Numéro 9|2007, mis en ligne le 2 février 2009, consulté le 2 novembre 2018, [http://journals.openedition.org/rhei/2543]; DOI: $10.4000 /$ rhei.2543.

4. Ainsi qu'une exposition virtuelle mise en ligne sur le site Criminocorpus : "Les détenus mineurs de la maison d'Éducation surveillée de Fresnes, 1929-1958 ", Musée Criminocorpus, publié le 8 mars 2019, consulté le 18 mars 2019, [ https:// criminocorpus.org/fr/ref/25/19128/] 
5. Le prénom et le nom ont été changés. Archives départementales du Val-de-Marne (ADVM), 511W 381.

6. MODIANO Patrick, Dora Bruder, Paris, Gallimard, 1999.

7. Seine, Prisons de Fresnes, rapport au ministre de la Justice, Inspection générale des services administratifs, 12 décembre 1936, Archives nationales (AN), F1a 4577.

8. Carnet médical, 1949, ADVM, 511 W 381.

9. Enquête sociale, décembre 1948, ADVM, 511W 381.

10. Ibid.

11. Greffe judiciaire central, extrait du registre d'écrou, 2 mars 1949, ADVM, 511W 381.

12. Savigny-sur-Orge, fiche de signalement, 14 octobre 1948, ADVM, 511W 381.

13. Copie du rapport fourni par l'inspecteur Dessaint transmis au directeur du centre d'observation des mineurs de Savigny-sur-Orge, 6 novembre 1948, ADVM, 511W 381.

14. Extrait du jugement du tribunal pour enfants de Versailles, $1^{\text {er }}$ mars 1949, ADVM, 511W 381.

15. Je remercie Franciele Becher pour m'avoir transmis cette information.

16. Service social d'Aide aux Émigrants, 7 juillet 1950, ADVM 511W 389.

17. ADVM 511W389.

18. Entretien d'arrivée, ministère de la Justice, Direction de l'Éducation surveillée, centre d'observation de mineurs délinquants de Paris, ADVM 511W 389.

19. Ibid.

20. Savigny-sur-Orge, Examen psychiatrique, 7 juillet 1950, ADVM 511W 389.

21. PÉRISSOL Guillaume, "Les inventions de John. Un dossier de vagabond du tribunal pour enfants de la Seine (1945-1949) ", in BANTIGNY Ludivine, VIMONT Jean-Claude (dir.), Sous l'œil de l'expert: les dossiers judiciaires de personnalité, Mont-Saint-Aignan, Publications des Universités de Rouen et du Havre, 2010, p.53-68. Je remercie Guillaume Périssol pour m'avoir transmis ce texte.

22. Selon leurs fiches conservées au Centre d'exposition " Enfants en justice », Roman a séjourné à Savigny-sur-Orge du 29 avril 1948 au 10 novembre 1948 et Johann du 14 septembre 1948 au 4 octobre 1948. Je remercie Danièle Brière pour m'avoir transmis cette information.

\section{RÉSUMÉS}

Les archives départementales du Val-de-Marne conservent les dossiers de mineurs incarcérés à la maison d'éducation de Fresnes qui fut en activité de 1929 à 1958, date à partir de laquelle elle devint un centre spécial d'observation de l'Éducation surveillée. Les prévenus y étaient l'objet d'une procédure d'évaluation conduite par des agents de l'établissement chargés de rédiger un dossier sur la base duquel le juge pour enfants pouvait ensuite se baser pour prononcer sa décision. Parmi ceux-ci figure celui de Jean Gregg qui présente le profil d'un jeune homme 
victime du contexte de la Seconde Guerre mondiale et qui déroule plusieurs versions de son existence aux agents chargés de son observation. Cette stratégie vise à brouiller les pistes de la vie d'un jeune Allemand condamné plusieurs fois pour infraction à la police sur les étrangers, et qui cherche à fuir son foyer pour gagner l'Amérique, symbole d'une promesse d'adoption sans lendemain que lui aurait formulée un jeune soldat américain.

The Departmental Archives of Val-de-Marne keep records of juveniles incarcerated at Fresnes Education House from 1929 to 1958, from which date it became a Special Observation Center for Supervised Education. The defendants were the subject of an evaluation procedure conducted by officers of the prison who completed a file on the basis of which the juvenile judge could then base his decision. Among these is Jean Gregg who presents the profile of a young man victim of the context of the Second World War and who tells several versions of his existence to the agents in charge of his observation. This strategy aims to confuse the tracks of a young German man who has been sentenced several times for police offenses against foreigners, and who is trying to flee his home to America, a vain promise of adoption supposedly formulated by a young American soldier.

\section{INDEX}

Mots-clés : justice des mineurs, dossiers de jeunes, récit de vie, incarcération des mineurs Keywords : juvenile justice, case files, life story, juvenile detention

\section{AUTEUR}

\section{JEAN-LUCIEN SANCHEZ}

Docteur en histoire, Jean-Lucien Sanchez est chargé d'études en histoire au Laboratoire de recherche et d'innovation de la Direction de l'administration pénitentiaire. Il est membre correspondant du Centre pour les humanités numériques et l'histoire de la justice (CLAMOR, UMS CNRS 3726) et chercheur associé au Centre de recherches sociologiques sur le droit et les institutions pénales (CESDIP, UMR CNRS 8183). Il est membre du comité de rédaction de la revue Criminocorpus et auteur de l'ouvrage À perpétuité. Relégués au bagne de Guyane (Vendémiaire, 2013). 